\title{
APOSTAR POR LA CALIDAD DE LA EDUCACIÓN SUPERIOR A DISTANCIA DESDE LA PERTINENCIA SOCIAL
}

\author{
A NEW PROPOSAL TO QUALITY FORM THE POINT OF VIEW OF RELEVANCE OF DISTANCE \\ HIGHER EDUCATION
}

María José Rubio Gómez, Luis Miguel Romero Fernández, Universidad Técnica Particular de Loja (Ecuador)

\section{RESUMEN}

Considerando el gran interés que la educación a distancia ha despertado en los últimos años en Latinoamérica y el Caribe nos surgen diversas preocupaciones desde la necesidad y la pertinencia de la misma, entre otras, la constatación de que en muchos países aún no existe una regulación oficial de esta modalidad de educación, mucho menos aún, existen sistemas oficiales para evaluar y acreditar su calidad. Presentamos el estudio de la reglamentación de la educación a distancia y de los procesos de evaluación y acreditación de la calidad según los diferentes países de Latinoamérica y el Caribe, así como la propuesta para el desarrollo de dichos procesos a través del Instituto Latinoamericano y del Caribe de Calidad en Educación Superior a Distancia (CAL-ED).

\begin{abstract}
Considering the profound interest aroused by distance education in Latin America and the Caribbean of late years, different concerns about the necessity and relevance of distance education emerge. For example, the fact that many countries do not have official regulations for this educational system neither they have official systems for the assessment and accreditation of its quality. We are presenting the study of the regulations of distance education and the quality assessment and accreditation processes according to different countries in Latin America and the Caribbean. Moreover the proposal for the development of such processes through the "Instituto Latinoamericano y del Caribe de Calidad en Educación Superior a Distancia” (Latin American and Caribbean Institute for Quality in Distance Higher Education) (CALED)will be presented.
\end{abstract}




\section{SITUACIÓN ACTUAL}

El constante proceso de transformación de las sociedades contemporáneas exige a las organizaciones educativas y a los sistemas a los cuales pertenecen, dar respuestas adecuadas a los requerimientos de cada circunstancia y a los entornos en los que se desarrollan.

En este caso la necesidad de una educación permanente para los pueblos del primer mundo y, asimismo, una educación superior que pueda estar al alcance de todos, en los pueblos del tercer mundo; hoy, no se pone en duda.

Por ello, no es nada extraño considerar que el siglo XXI es el siglo de la educación permanente, es el siglo del conocer desde la educación y podemos también sostener, que la estrategia de formación más adecuada para tales actividades es la educación a distancia (EaD), por la flexibilidad que nos ofrece para simultanear el trabajo, el estudio y las obligaciones familiares; sin tener que concurrir a una sala de clase. $\mathrm{Si}$ hablamos de pertinencia, nada más pertinente que la $\mathrm{EaD}$, si consideramos este término según el Diccionario de la Real Academia Española: es aquello de lo que se dice viene a propósito, es adecuado o conveniente.

De ahí, el gran interés que ha despertado esta modalidad de enseñanzaaprendizaje en universidades y empresas, que han visto en ella una herramienta valiosa para brindar capacitación, actualización y profesionalización a una población cada vez más numerosa con demandas y necesidades de formación diversificadas.

En estos últimos años, la incorporación delas nuevas tecnologías de la información y la comunicación (TICs), han enriquecido enormemente a la EaD abriendo espacios para la interacción, flexibilidad, inmediatez en la actualización de los contenidos y en la asesoría a los alumnos, permitiendo acortar distancias.

La pertinencia a su vez nos interroga y se entrecruza con el concepto de calidad ya que las instituciones de educación deben dar una respuesta conforme a los requerimientos de la sociedad desde la calidad y cualidad deseadas, y mucho más en este momento donde la competitividad se impone. La pertinencia hace referencia a la capacidad de la educación superior de dar respuestas oportunas y significativas a cada problema o a cada situación particular.

Así, la pertinencia y la calidad en la EaD se complementan ante la necesidad actual de la educación superior. La calidad es un concepto propio de las instituciones universitarias desde su origen y en relación con la sociedad, por lo tanto, debe ser 
María José Rubio y Luis Miguel Romero

Apostar por la CAlidad de la EduCACión SuPERIor a Distancia desde la Pertinencia Social

asumida contextualmente y no como estándar uniformante. Interesarse por una mayor pertinencia social y elevar la calidad de las instituciones, programas y procesos de la educación superior implica repensar la organización universitaria siendo más flexibles y sobre todo ampliando sus horizontes y las posibilidades de relacionarse.

La evaluación y la posterior acreditación institucional y de programas, servirá para constatar la pertinencia social y la calidad, permitiendo la necesaria vinculación entre instituciones con confianza mutua.

Promover un acercamiento de las universidades a las comunidades desfavorecidas a través de la educación a distancia, así como el acercamiento a los sectores que no pueden acceder a una educación presencial, habla de la capacidad de estas instituciones para intervenir positivamente en la búsqueda de soluciones a los problemas que confrontan dichas comunidades o sectores sociales.

El tema de la calidad en la educación superior, ha venido cobrando especial vigencia en la multiforme realidad universitaria mundial, y fue junto con la pertinencia, el núcleo de la reflexión de la última conferencia mundial de la UNESCO sobre Educación Superior; así ambos conceptos, pertinencia de la educación a distancia y calidad son hoy en día los dos núcleos de mayor interés, y también de preocupación, en la realidad universitaria latinoamericana.

$\mathrm{Al}$ reflexionar sobre las tendencias actuales en educación abierta y a distancia en Latinoamérica y el Caribe, se concluye que la proliferación de programas y títulos en diversas instituciones no controlados en su calidad, ha hecho mucho daño a la EaD ofrecida desde el rigor académico y de servicio; por ello, la reciente preocupación por la evaluación de la calidad creando estándares para la EaD es un acierto para saber en que terreno nos movemos. La mejor forma de evaluar la importancia y efectividad de cualquier tipo de institución educativa es seleccionar los indicadores y estándares más adecuados relativos a la calidad, la innovación y la pertinencia de la institución en general y de los programas en particular.

Las universidades que ofertan programas en Educación a Distancia, lo quieran o no, deben demostrar que sus programas son de calidad, el mundo en el que vivimos así lo demanda y la universidad no puede ser una isla en este u otros aspectos, más bien debe de ir por delante en todo aquello que ayude al progreso y a la mejora de los servicios y de la calidad de vida de los seres humanos. Es por esta razón que todas las instituciones caminan hacia la evaluación y la acreditación. 
$\mathrm{Al}$ hablar de calidad y acreditación en las universidades no podemos olvidarnos de los procesos rápidos de internacionalización, y como ha contribuido y contribuye, a este aspecto, la educación a distancia. Son muchas las universidades que han extendido sus servicios más allá de las fronteras de los países donde nacieron y esto provoca nuevas inseguridades por la calidad de lo desconocido.

Necesitamos tener la certeza que los programas que vienen de fuera están garantizados, de la misma forma que lo conocido, es una exigencia que nos interpela con urgencia para dar solución a las inquietudes que nos planteamos ante ofertas educativas a nivel internacional o ante la implantación de diversas instituciones con sedes en varios países.

Otras tendencias actuales son la oferta conjunta de programas a distancia a través de alianzas estratégicas entre varias universidades, y lo que se conoce como organizaciones corporativas; al igual que la movilidad de la población estudiantil y docente que nos lleva a la necesidad de la evaluación y acreditación de la EaD.

Frente a la proliferación de modelos de evaluación y acreditación, es necesario tener en cuenta las iniciativas consensuadas de estos procesos con el fin de atender todos los aspectos señalados anteriormente. En este sentido hemos de destacar los logros de consenso surgidos a través del modelo del Proyecto del Banco Interamericano de Desarrollo (BID) "Centro Virtual para el Desarrollo de Estándares de Calidad para la Educación Superior a Distancia en América Latina y el Caribe", que estuvo auspiciado también por la Asociación Iberoamericana de Educación Superior a Distancia (AIESAD) y el Consorcio Red de Educación a Distancia (CREAD) participando como Entidad Ejecutora del mismo la Universidad Técnica Particular de Loja (UTPL) - Ecuador. A pesar de la amplia participación y altos niveles de consenso y difusión del proyecto, con excepción de algunas instituciones que tomaron de base el citado trabajo, se han seguido investigando en diversos sistemas de estándares por parte de numerosas instituciones, algunas privadas y con ánimo de lucro; en otras palabras, se ha seguido el mito de trabajar en enmiendas a la totalidad para ser originales, no sólo con lo que supone de inversión de cuantiosas energías institucionales, sino sobre todo por la dispersión y confusión que se ha generado. Por todo ello, teniendo en cuenta la aceptación y los esfuerzos realizados, en este trabajo ampliamente consensuado; por parte de la Universidad Técnica Particular de Loja se continua trabajando en el mismo, junto con otras instituciones, con el fin de aportar un Modelo de Evaluación valido, aceptado internacionalmente y conocido por las instituciones y expertos que lo avalan. 
María José Rubio y Luis Miguel Romero

Apostar por la CAlidad de la EduCACión SuPERIor a Distancia desde la Pertinencia Social

\section{NECESIDAD DE LA REGLAMENTACIÓN DE LA EDUCACIÓN SUPERIOR A DISTANCIA Y DE LOS PROCESOS DE EVALUACIÓN DE LA CALIDAD. INICIATIVAS NACIONALES DE LA REGIÓN}

Las dos preocupaciones fundamentales en este momento en relación a la EaD Latinoamérica y el Caribe son:

- $\quad$ La reglamentación por parte de los diferentes países que dé validez a los programas de EaD

- La calidad y acreditación de la Educación a Distancia.

Frente a la necesidad de mejorar la calidad de la Educación Superior en general, los gobiernos de la mayoría de los países latinoamericanos y caribeños han desarrollado importantes iniciativas con la creación de organismos competentes que realicen los procesos de evaluación y acreditación, pero que han tenido muy escasa repercusión en relación a la EaD.

En Latinoamérica se ha conformado la Red Iberoamericana para la Acreditación de la Calidad de la Educación Superior (RIACES), constituida formalmente en Buenos Aires en mayo de 2003, como asociación de agencias y organismos de evaluación y acreditación de la calidad de la educación superior. La Red es independiente de cualquier Estado y sus miembros han de tener competencias reconocidas por sus respectivos gobiernos o Estados en esta materia. Los fines de la Red son promover entre los países iberoamericanos la cooperación y el intercambio en materia de evaluación y acreditación de la calidad de la educación superior. Es de destacar que entre sus fines no está la acreditación internacional, ni específicamente la evaluación y acreditación de la Educación a Distancia, sino, fortalecer los vínculos entre las agencias nacionales de acreditación.

Los países e instituciones que conforman la RIACES son: ARGENTINA Ministerio de Educación, Ciencia y Tecnología; Comisión Nacional de Evaluación y Acreditación Universitaria (CONEAU). BOLIVIA: Ministerio de Educación. BRASIL: Instituto Nacional de Estudos e Pesquisas Educacionais Anísio Teixeira (INEP). CHILE: Comisión Nacional de Acreditación de Pregrado (CNAP). COLOMBIA: Ministerio de Educación; Consejo Nacional de Acreditación (CNA). COSTA RICA: Sistema Nacional de Acreditación de la Educación Superior (SINAES). CUBA: Junta de Acreditación Nacional (JAN). ECUADOR: Consejo Nacional de Evaluación y Acreditación de la Educación Superior (CONEA). EL SALVADOR: Ministerio de Educación de El Salvador. ESPAÑA: Agencia Nacional de Evaluación de la Calidad y Acreditación (ANECA). MÉXICO: Consejo para la Acreditación de la Educación 
Superior (COPAES). NICARAGUA: Consejo Nacional de Universidades (CNU). PANAMÁ: Universidad de Panamá. PARAGUAY: Agencia Nacional de Evaluación y Acreditación de la Educación Superior (ANEAES). PERÚ: Asamblea Nacional de Rectores (ANR). REPÚBLICA DOMINICANA: Secretaria de Estado de Educación Superior, Ciencia y Tecnología. URUGUAY: Ministerio de Cultura y Educación. VENEZUELA: Sistema de Evaluación Académica (SEA). Consejo Superior Universitario Centroamericano (CSUCA). Consejo Centroamericano de Acreditación (CCA). Consejo Universitario Iberoamericano (CUIB). Instituto Internacional para la Educación Superior en América Latina y el Caribe (IESALC-UNESCO). Organización de Estados Iberoamericanos para la Educación, la Ciencia y la Cultura (OEI).

\section{Reglamentación en Educación a Distancia y procesos de evaluación de la calidad según los diferentes países de Latinoamérica y el Caribe:}

\section{ARGENTINA}

\section{Reglamentación:}

- Decreto Reglamentario de la Ley de Educación Superior, con el tema "UNIVERSIDADES -MODALIDADES EDUCATIVAS -EDUCACIÓN A DISTANCIA -MINISTERIO DE CULTURA Y EDUCACIÓN"

- Res. Min. $\mathrm{N}^{0}$ 1716/98 De la fijación de normas y pautas mínimas para la modalidad "educación a distancia". Deroga la Res. Min. $\mathrm{N}^{\circ}$ 1423/98.

- El Ministerio de Educación Ciencia y Tecnología mediante resolución No. 1717 presenta los "Lineamientos para la presentación y evaluación de programas y carreras bajo la modalidad de educación a distancia"

- Res. Min. $\mathrm{N}^{0}$ 236/01 Del reconocimiento oficial de los títulos de postgrado de carreras de modalidad educativa "no presencial" o "a distancia”.

\section{Acreditación}

- Ley de Educación Superior No. 24.521 en la sección EVALUACION Y ACREDITACION (artículos 44 al 47)

- Decreto nacional 173/96. Decreto Reglamentario dela ley de Educación Superior con el tema "UNIVERSIDADES-COMISION NACIONAL DE EVALUACION Y ACREDITACION UNIVERSITARIA” 
María José Rubio y Luis Miguel Romero

Apostar por la Calidad de la Educación Superior a Distancia desde la Pertinencia Social

- La resolución No. 1168/97 establece los estándares y criterios a aplicar a los procesos de acreditación de carreras de postgrado dejando expresa constancia de que dichos criterios son de aplicación exclusiva a la modalidad de educación "presencial", difiriendo el tratamiento de las pautas para la modalidad "no presencial" o "a distancia", para cuando se cuente con la reglamentación pertinente...

\section{BOLIVIA:}

\section{Reglamentación:}

- No se ha podido detectar si existe alguna reglamentación específica en educación a distancia.

\section{Acreditación}

- Ley del Consejo Nacional de Acreditación de Educación Superior, en la cual se señala la creación del Consejo Nacional de Acreditación de la Educación Superior (CONAES)

- ElCONAES es la institución oficial y de carácter público de acreditación de la República están bajo su responsabilidad la acreditación de la calidad de los programas académicos y de las Instituciones de Educación Superior, así como la coordinación, supervisión y ejecución de los procesos de evaluación externa conducentes a la acreditación.

BRASIL

\section{Reglamentación}

- Decreto $\mathrm{N}^{0}$ 5.622, de 19 de diciembre de 2005, en el cual se dan las directrices para la Educación a Distancia

\section{Acreditación}

- El Ministro de Estado de Educación en la Circular $\mathrm{N}^{0} 4.361$, de 29/12/2004 resuelve sobre los procesos de acreditación de instituciones de educación superior, acreditación para oferta de cursos de postgrado, y acreditación de instituciones de educación superior para oferta de cursos superiores a distancia. 
María José Rubio y Luis Miguel Romero

Apostar por la Calidad de la Educación Superior a Distancia desde la Pertinencia Social

- La Secretaria de Educación a Distancia ha elaborado el documento "Referencias de calidad para cursos a distancia" con el fin de orientar a las Comisiones que analizan los proyectos de cursos a distancia.

\section{CHILE:}

\section{Reglamentación:}

- En Chile no existe una ley exclusiva sobre educación superior. La Ley $\mathrm{N}^{\circ} 18.962$ Orgánica Constitucional de Enseñanza (LOCE), publicada en Diario Oficial de 10 de marzo de 1990, y que ha experimentado modificaciones en los años 1991, 1998, 2001, 2003 y 2004; aporta las bases generales respecto de la educación en sus niveles parvulario, básico, medio y superior, planteando respecto de este último el sistema general de educación superior vigente el cual se complementa con varias normas posteriores.

\section{Acreditación:}

- En marzo de 1999 se constituyó la Comisión Nacional de Acreditación (CNAP). El CNAP cuenta con una "Ley que establece un sistema nacional de aseguramiento de la Calidad de la Educación Superior"

- En septiembre de 1999 se creó la Comisión Nacional de Acreditación de Postgrado (CONAP). El CONAP cuenta con un Decreto que establece las "Normas y Procedimientos para la Evaluación Experimental de la Calidad de los Programas de Postgrado de las Universidades Autónomas

No se especifica procesos específicos para la evaluación de la calidad en EaD.

\section{COLOMBIA}

\section{Reglamentación:}

- "La ley 30 de 1992, en su artículo 15 determina la posibilidad de ofertar programas utilizando la metodología de educación a distancia: 
María José Rubio y Luis Miguel Romero

Apostar por la Calidad de la Educación Superior a Distancia desde la Pertinencia Social

Las instituciones de Educación Superior podrán adelantar programas en la metodología de educación abierta y a distancia, de conformidad con la presente Ley.

Actualmente no se ha reglamentado nada en particular, quedando como una especie de vacío conceptual y jurídico que le dé alguna identidad al trabajo que se desarrolla en la Educación a Distancia. Sin embargo, se ha venido presentando y trabajando en una propuesta conceptual para el Decreto Reglamentario de los Estándares Mínimos de Calidad para la oferta de programas en esta modalidad. Desde el punto de vista jurídico, la Educación a Distancia, sea que utilice las estrategias tradicionales de esta modalidad o utilice las TIC en lo que se llama enseñanza virtual, sigue siendo una metodología."

\section{Acreditación}

- El CNA ha desarrollado una versión preliminar de la guía "Indicadores para la autoevaluación con fines de acreditación de programas de pregrado en las modalidades a distancia y virtual"

\section{COSTA RICA}

\section{Reglamentación:}

- La Asamblea Legislativa de la Republica de Costa Rica decreta la Ley de Creación de la Universidad Estatal a Distancia (UNED) No. 6044. Publicada en "La Gaceta" No.50 de 12 de marzo de 1977

\section{Acreditación:}

- La Asamblea Legislativa de la República de Costa Rica decreta la creación de la Ley del Sistema Nacional de Acreditación de la Educación Superior (SINAES) el 17 de mayo del 2002

- El SINAES ha elaborado un Adendum al Manual de acreditaciónConvocatoria Año 2000 para atender la acreditación oficial de carreras universitarias con la modalidad de educación a distancia con lineamientos mínimos. 


\section{CUBA}

\section{Reglamentación}

- "No existe una ley orgánica de Educación Superior. El Ministerio de Educación Superior MES para ejecutar la política del Gobierno en materia de Educación Superior dicta reglamentos y disposiciones, atendiendo a su carácter normativo y metodológico"3.

- La Universidad de la Habana imparte educación a distancia desde 1979

- Actualmente se está poniendo especial interés en programas a distancia y semipresenciales.

\section{Acreditación:}

- La Junta de Acreditación Nacional (JAN) representa a la Educación Superior cubana en materia de calidad, evaluación y acreditación ante las agencias, instituciones y organizaciones de calidad, evaluación y acreditación de los diferentes países o regiones, o de carácter internacional.

- En Julio del 2004 editó el Reglamento del Sistema de Evaluación de Maestrías (SEA-M), las cuales se puede acreditar como programas Autorizados, Ratificados, Certificados o Programas de Excelencia. No se hace alusión a una evaluación diferente para presencial o a distancia.

\section{ECUADOR}

\section{Reglamentación}

- La Educación Superior en el Ecuador la rige el Consejo Nacional de Educación Superior (CONESUP),

En la Ley de Educación Superior se expresa:

"Art.3. Las instituciones del Sistema Nacional de Educación Superior ecuatoriano, en sus diferentes niveles, tienen los siguientes objetivos y Estrategias fundamentales: 
María José Rubio y Luis Miguel Romero

Apostar por la Calidad de la Educación Superior a Distancia desde la Pertinencia Social

a) Formar, capacitar, especializar y actualizar a estudiantes y profesionales en los niveles de pregrado y postgrado, en las diversas especialidades y modalidades.

Art.13.- Son atribuciones y deberes del CONESUP:

f) Aprobar la creación de extensiones y programas de postgrado, así como fijar los lineamientos generales para las modalidades de educación semipresencial y a distancia, que deberán acreditar condiciones y niveles de calidad similares a los de la educación presencial;

p) Normar el funcionamiento de los cursos de postgrado"

- El CONESUP ha dado algunas líneas generales para aprobación de carreras de pregrado y de postgrado en Educación a Distancia, pero aún están pendiente de aprobación los lineamientos que se indican en la ley.

\section{Acreditación}

- El Consejo Nacional de Evaluación y Acreditación (CONEA), del cual la UTPL forma parte de la comisión de evaluación a distancia y virtual, se encuentra desarrollando la guía de evaluación de programas de educación a distancia, tomando como base los estándares desarrollados en el proyecto "Centro Virtual para el Desarrollo de Estándares de Calidad para la Educación Superior a Distancia en América Latina y el Caribe”.

\section{EL SALVADOR}

\section{Reglamentación}

- No se ha podido detectar si existe alguna reglamentación específica en educación a distancia.

\section{Acreditación}

- Ley de Educación Superior (diciembre 1995) COMISION DE ACREDITACION 
María José Rubio y Luis Miguel Romero

Apostar por la Calidad de la Educación Superior a Distancia desde la Pertinencia Social

"Art. 41.- Crease la Comisión de Acreditación de la calidad académica como un ente adscrito al Ministerio de Educación con la función de aplicar el sistema de acreditación académica.

Art. 42.- El proceso de acreditación será la evaluación continúa que utilizará la Comisión de Acreditación para calificar la calidad académica de aquellas instituciones que llenen los requisitos establecidos en la presente Ley y que voluntariamente lo soliciten”.

\section{MÉXICO}

\section{Reglamentación}

- La Asociación Nacional de Universidades y Escuelas Politécnicas (ANUIES) entre sus documentos estratégicos contiene el Plan Maestro de Educación Superior Abierta y a Distancia. Líneas estratégicas para su desarrollo, impreso en octubre de 2001.

- La ANUIES además ha elaborado el documento Elementos Normativos a considerar para los Programas de Educación Superior Impartidos en Modalidades Alternativas a la Escolarizada

- $\quad$ Red Nacional de Educación Superior a Distancia. La colaboración permanente entre instituciones y regiones es una estrategia fundamental para consolidar la educación a distancia como una modalidad innovadora. Bajo esta premisa en el año 2000 se inicia la constitución de nodos regionales de educación a distancia, conformados en cada uno de los Consejos Regionales de la ANUIES.

Los nodos regionales tienen como labor fundamental abordar de manera articulada los aspectos académicos, administrativos y de soporte tecnológico para la educación a distancia en cada región. Sus acciones de colaboración permanente, fundamentadas en propósitos y visiones comunes, permiten consolidar la Red Nacional de Educación Superior a Distancia.

Esta Red está integrada por los siguientes nodos:

- $\quad$ Red de Educación Abierta y a Distancia del Noroeste (READIN)

- Red de Educación a Distancia de la Región Noreste 
María José Rubio y Luis Miguel Romero

Apostar por la Calidad de la Educación Superior a Distancia desde la Pertinencia Social

- Red de Educación a Distancia de la Región Centro Occidente

- Red de Educación Abierta y a Distancia de la Región Sur Sureste

- Red de Educación a Distancia de la Región Metropolitana

- Red de Educación a Distancia de la Región Centro Sur

Con el uso de las tecnologías, cada uno de estos grupos de trabajo ha emprendido acciones de diagnóstico, cooperación, intercambio, formación de recursos humanos, estudios y difusión, lo cual permite avanzar en el desarrollo de la modalidad.

\section{Acreditación}

- El Consejo para la Acreditación de la Educación Superior, A. C. (COPAES), fundado el 24 de octubre de 2000, es la única instancia validada por la Secretaria de Educación Publica para conferir reconocimiento oficial a los organismos acreditadores de los programas académicos que se imparten en este nivel educativo en México. Los organismos acreditadores reconocidos por el COPAES están facultados para llevar a cabo los procesos de evaluación conducentes a la acreditación de programas de nivel de licenciatura y de técnico superior universitario o profesional asociado, en áreas definidas del conocimiento, en las instituciones públicas y privadas de todo el país.

- La Federación de Instituciones Mexicanas Particulares de Educación Superior (FIMPES) es una asociación civil de afiliación voluntaria que acredita a instituciones particulares de educación superior que han alcanzado altos estándares de calidad. Entre las convicciones fundamentales de la Federación, está la referente a que el nivel de desarrollo de un país depende sustancialmente de la calidad de la educación que en él se ofrece.

Dentro de los criterios de Acreditación que aplica FIMPES el capítulo XI se refiere a Educación a Distancia.

Los Comités Interinstitucionales para la Evaluación de la Educación Superior (CIEES) son nueve cuerpos colegiados, integrados por pares académicos del más alto nivel de las instituciones de educación superior de todo el país. Su misión fundamental es evaluar las funciones y los programas académicos que se imparten en las 
instituciones educativas que lo solicitan y formular recomendaciones puntuales para su mejoramiento, contenidas en los informes de evaluación, que se entregan a los directivos de las instituciones. En el caso de la educación a distancia han elaborado una Tabla Guía de Autoevaluación Modalidad Mixta y no Escolarizada que contiene algunos parámetros propios para dicha modalidad. ${ }^{4}$

- Consejo Nacional de Ciencia y Tecnología CONACYT. Con el propósito de coadyuvar al logro de los objetivos estratégicos planteados en el Programa Nacional de Educación (PNE) y el Programa Especial de Ciencia y Tecnología (PECYT) 2001-2006, la SEP y el CONACYT establecieron entre sus líneas de acción la creación, en el año 2001, del Programa para el Fortalecimiento del Postrado Nacional (PFPN). Uno de los componentes del PFPN es el Padrón Nacional de Postrado (PNP) que, establecido en 2002, representa en la actualidad el marco de referencia para el diseño y fortalecimiento de los programas educativos de postgrado en todos sus niveles y orientaciones.

\section{NICARAGUA}

\section{Reglamentación:}

- En la Ley General de Educación de dicho país en su Art.6: Definiciones Generales que expresan el enfoque de la educación nicaragüense, se manifiesta:

"Educación a Distancia: la educación a distancia se diseña generalmente para atender a los estudiantes que por diversas razones no pudieron asistir a la educación regular y a través de los recursos tecnológicos existentes se producen los procesos de enseñanza y aprendizaje, mediados siempre por el maestro. Generalmente se acompañan de enseñanza Radiofónica, Televisiva, Medios Impresos, enseñanza virtual, etc".

\section{Acreditación}

- Se ha realizado un primer taller de evaluación y acreditación. Los objetivos de este taller son contribuir al desarrollo de una cultura de calidad en las instituciones miembros del Consejo Nacional de 
María José Rubio y Luis Miguel Romero

Apostar por la Calidad de la Educación Superior a Distancia desde la Pertinencia Social

Universidades y fomentar la articulación del trabajo de las unidades técnicas de evaluación que contribuya a sentar las bases para el Sistema Nacional de Evaluación y Acreditación en Nicaragua.

\section{PANAMÁ}

\section{Reglamentación}

- "La educación a distancia, en el marco legal, no posee normativa que se refiera a ella como modalidad educativa propiamente dicha. La Ley 47 Orgánica de Educación, contempla esta metodología como perteneciente a la sección segunda de la educación para jóvenes y adultos en cualquiera de sus tres niveles: educación básica, educación media y tercer nivel.

En la legislación se consigna la responsabilidad del Estado de ofrecer servicios educativos a poblaciones tradicionalmente marginadas, como las indígenas y penitenciarias. Se logra, además, consignas de apertura para el uso de técnicas innovadoras, en atención a las características biosicosociales del adulto.

Por otro lado, las instituciones que ofrecen el servicio educativo a distancia a nivel superior, y más específicamente educación a nivel universitario, son instituciones privadas instituidas bajo el Decreto Ley 16 de 11 de junio de 1963, el cual reconoce el derecho de crear y hacer funcionar en la República de Panamá, las universidades privadas.

Es la Universidad de Panamá la institución encargada, por disposición constitucional, de aprobar los planes y programas de estudios de las Universidades privadas y fiscalizar el quehacer de dichas instituciones para garantizar la calidad de los títulos que se expiden" 5

\section{Acreditación}

- El 15 de julio de 1997 se creo el Consejo Nacional de Acreditación como el Organismo de Acreditación autorizado por el Estado. 


\section{PARAGUAY}

\section{Reglamentación}

- En la Ley General de Educación se indica: DE LA EDUCACIÓN A DISTANCIA:

“Artículo 59: Se extenderá el acceso a la educación en todos sus niveles a personas que por sus condiciones de trabajo, su ubicación geográfica su impedimento físico o de edad no pueden asistir a las instituciones de educación formal. El Ministerio de Educación y Cultura promoverá el uso de los medios previstos por la tecnología de las comunicaciones a distancia.

La autoridad competente de las telecomunicaciones reservará frecuencias de radio, de televisión por aire, por cable u otro medio similar para desarrollar iniciativas de educación a distancia.

Artículo 60: El Gobierno promoverá y apoyarála educación a distancia de iniciativa privada y reglamentará el currículo, los programas y el sistema de evaluación, para el reconocimiento oficial de los cursos y actividades impartidas y de sus respectivos certificados y títulos".

\section{Acreditación}

- Ley General de Educación: DE LA CALIDAD DE LA EDUCACIÓN Y SU EVALUACIÓN:

"Artículo 20: El Ministerio de Educación y Cultura, las gobernaciones, los municipios y las comunidades educativas, garantizarán la calidad de la educación. Para ello se realizará evaluación sistemática y permanente del sistema y los procesos educativos.

Artículo 21: Las instituciones educativas públicas y privadas otorgarán a las autoridades educativas facilidades y colaboración para la evaluación.

Artículo 22: Las autoridades educativas darán a conocer a los maestros, alumnos, padres de familia y a la sociedad en general, los resultados de las evaluaciones que realicen, así como las informaciones globales que permitan medir el desarrollo y los avances de la educación". 
María José Rubio y Luis Miguel Romero

Apostar por la Calidad de la Educación Superior a Distancia desde la Pertinencia Social

- La Agencia Nacional de Evaluación y Acreditación de la Educación Superior (ANEAES) fue creada por Ley 2072/03, sancionada por el Congreso de la Nación Paraguaya, a los trece días del mes febrero del año dos mil tres. Depende del Ministerio de Educación y Cultura, pero goza de autonomía técnica y académica para el cumplimiento de sus funciones.

No se reglamenta específicamente nada sobre la calidad de la educación a distancia.

\section{PERÚ}

\section{Reglamentación:}

- La Ley General de Educación $\mathrm{N}^{\circ} 28044$ en su Artículo $27^{\circ}$ sobre La Educación a Distancia indica: "La Educación a Distancia es una modalidad del Sistema Educativo caracterizada por la interacción simultánea o diferida entre los actores del proceso educativo, facilitada por medios tecnológicos que propician el aprendizaje autónomo. Es aplicable a todas las etapas del Sistema Educativo, de acuerdo con la normatividad en la materia.

Esta modalidad tiene como objetivo complementar, reforzar o reemplazar la educación presencial atendiendo las necesidades y requerimientos de las personas. Contribuye a ampliar la cobertura y las oportunidades de aprendizaje".

\section{Acreditación}

- Corresponde al Estado garantizar los factores de calidad en las instituciones públicas, en las instituciones privadas las regula y supervisa. La Ley mencionada 28044, en su artículo 14 establece un "Sistema Nacional de Evaluación, Acreditación y Certificación de la Calidad Educativa que abarca todo el territorio nacional y responde con flexibilidad a las características y especificidades de cada región del país. El Sistema opera a través de organismos autónomos, dotados de un régimen legal y administrativo que garantiza su indenpendencia”. La Ley del Sistema Nacional de Evaluación, Acreditación y Certificación de la Calidad Educativa (SINEACE) No 28740 señala en el art. 1 "La presente Ley norma los procesos de 
María José Rubio y Luis Miguel Romero

Apostar por la Calidad de la Educación Superior a Distancia desde la Pertinencia Social

evaluación, acreditación y certificación de la calidad educativa, define la participación del Estado en ellos y regula el ámbito, la organización y el funcionamiento del Sistema Nacional de Evaluación, Acreditación y Certificación de la Calidad a que se refieren los articulos 14 y 16 de la Ley No 28044. Ley General de Educación”.

- La Asamblea Nacional de Rectores en el Pleno del o1 de febrero del 2002, acordó la designación de la Comisión Nacional de Rectores para la Acreditación Universitaria a fin de revisar y hacer propuestas de estándares de acreditación y evaluación de las Universidades Peruanas.

\section{REPÚBLICA DOMINICANA}

\section{Reglamentación}

- La Secretaría de Educación Superior Ciencia y Tecnología está trabajando en la reglamentación de Educación a Distancia, pero hasta el momento no hay una reglamentación específica.

\section{Acreditación}

- Existe una Asociación Dominicana para el Autoestudio y la Acreditación (ADAAC), que es una agencia privada, no lucrativa y de finalidad pública que fue fundada en 1987 para dar respuesta a las inquietudes acerca de la necesidad de establecer algún mecanismo de autorregulación y mejoramiento de la calidad de la educación superior en el país.

- La Asociación Dominicana de Rectores de Universidades (ADRU) ha elaborado un pequeño sistema de indicadores de calidad de la educación superior dominicana, pero no señala nada sobre la evaluación de la educación superior a distancia.

\section{URUGUAY}

\section{Reglamentación}

- "No existe una "Ley de educación superior" que regule a este nivel en su conjunto. Además de los artículos de la Constitución de la 
María José Rubio y Luis Miguel Romero

Apostar por la Calidad de la Educación Superior a Distancia desde la Pertinencia Social

República .., las leyes más relevantes son tres: a) la ley orgánica de la Universidad de la República (UDELAR), número 12.549 (de 1958); b) la ley 15.661, que autoriza el funcionamiento de universidades privadas y el reconocimiento de sus títulos, de 1984; y c) la ley 15.739 (de 1985), que crea el Ente Autónomo de Administración Nacional de Educación Pública (ANEP), encomendándole a su Consejo Directivo Central la formación y el perfeccionamiento docente" ${ }^{\text {. }}$.

\section{Acreditación}

- Se ha aplicado el Mecanismo Experimental de Acreditación (MEXA) Se trata de un mecanismo que se aplicó por única vez, si bien es preparatorio de un procedimiento definitivo y permanente que se espera aplicar en el futuro. Algunos de los objetivos del ejercicio eran el conocimiento mutuo, el aprendizaje conjunto y la validación del mecanismo en los países y a nivel regional. El objetivo último es la mejora de la calidad en la enseñanza superior en nuestra región.

- Puesto que en Uruguay no se cuenta con una Agencia de acreditación, se conformaron Comisiones Ad Hoc designadas por el Poder Ejecutivo para cada carrera acreditada. La competencia de estas Comisiones se limitó al MEXA.

- El MEXA se planteó como un mecanismo experimental y muestral en la Región MERCOSUR. Esto significa que el principal objetivo operativo del ejercicio de acreditación era validar el mecanismo, más que realizar la acreditación masiva de carreras o establecer una categorización general de calidad.

\section{VENEZUELA}

\section{Reglamentación}

- En el Artículo 10 de la Ley de Universidades se indica: "Conforme a lo dispuesto en la Ley de Educación, el Ejecutivo Nacional, oída la opinión del Consejo Nacional de Universidades, podrá crear Universidades Nacionales Experimentales con el fin de ensayar nuevas orientaciones y estructuras en Educación Superior. Estas Universidades gozarán de autonomía dentro de las condiciones especiales requeridas por 
la experimentación educativa. Su organización y funcionamiento se establecerá por reglamento ejecutivo y serán objeto de evaluación periódica a los fines de aprovechar los resultados beneficiosos para la renovación del sistema y determinar la continuación, modificación o supresión de su status.

- Mediante Resolución No. 1600 - 16 de septiembre de 1996, Gaceta oficial de la República de Venezuela No. 5.098 Extraordinario, de fecha 19 de septiembre de 1996, de conformidad con los artículos 10 de la Ley de Universidades y 3 del Decreto No. 2.398 de fecha 27 de septiembre de 1977, se dicta el reglamento de la Universidad Nacional Abierta de Venezuela.

- El Consejo Consultivo Nacional de Postgrado (CCNPG) cuenta con unos lineamientos en los cuales se indica las características que deben tener los Programas de Postgrado de la Modalidad a Distancia

\section{Acreditación}

- El Sistema de Evaluación Académica (SEA) tendrá como propósitos fundamentales el aseguramiento e incremento de la calidad y el reconocimiento e incremento de la excelencia de las universidades nacionales. Esto supone que el objeto nodal de este sistema es la evaluación de la calidad universitaria.

\section{CONCLUSIONES}

Acorde a lo antes descrito, podemos concluir lo siguiente:

- $\quad$ La legislación en los países de la región contempla el funcionamiento específico de los programas de EaD en Argentina, Brasil, Costa Rica, Ecuador, Nicaragua, Panamá, Paraguay, Perú y Venezuela

- En varios de estos países hay iniciativas sobre lineamientos para autorizar el funcionamiento de los programas de EaD, como un control específico, que en la mayoría de los casos no se requiere para la educación presencial.

- Los organismos de evaluación y acreditación antes citados, preferentemente orientan su accionar a la evaluación de la educación presencial; como hemos visto hay muy pocas iniciativas oficiales que 
María José Rubio y Luis Miguel Romero

Apostar por la Calidad de la Educación Superior a Distancia desde la Pertinencia Social

contemplen la EaD, podría citarse el breve Adendum de Costa Rica con líneas claves para la acreditación de las carreras, la guía que está trabajando el CNA de Colombia, el trabajo que también está realizando el CONEA de Ecuador y, Brasil que ha elaborado las referencias de calidad para los cursos de EaD,

- En Bolivia, Chile, Cuba, El Salvador, Panamá, República Dominicana y Uruguay, no se ha podido detectar si existe alguna reglamentación específica en educación a distancia o sobre la calidad de la misma, sin embargo se podría interpretar que las universidades en algunos casos pueden ofrecer programas en EaD de acuerdo a la autonomía que se les confiere.

- Otro aspecto a destacar, en el ámbito de las estructuras de internacionalización, es el reconocimiento de títulos de universidades extranjeras, por una modalidad u otra, en la mayoría de los casos, debe ser a través de universidades nacionales.

\section{OTRAS INICIATIVAS DE EVALUACIÓN Y ACREDITACIÓN EN LA EDUCACIÓN SUPERIOR}

\section{Instituto Internacional para la Educación Superior en América Latina (IESALC)}

Ofrece un Curso de "Formación en Evaluación y Acreditación de la Educación Superior” en línea; además ofrece programas de software para la gestión universitaria fundamentadas en guías y manuales, realizados por especialistas en el área de la Educación Superior en diversos países de la región, pero ninguno de ellos está enfocado a la EaD.

1. "Sistema de Autoevaluación de Instituciones Universitarias Latinoamericanas y del Caribe (SAIULCA)"

2. "Sistema de Acreditación de Programas Académicos"

3. "Sistema de Autoevaluación de Instituciones Universitarias e Institutos de Educación Superior"

4. "Sistema de Evaluación de Currículo de Docentes"

5. "Sistema de Evaluación de Currículo de Pares Evaluadores"

6. "Sistema para la Autoevaluación de los Programas de Postgrado"

7. "Sistema de Pruebas de Autoevaluación IESALC"

8. "Sistema de Gestión de Matrícula SIGMA" 
María José Rubio y Luis Miguel Romero

Apostar por la CALidad de la Educación Superior a Distancia DESDE la Pertinencia Social

\section{Sistema Centroamericano de Evaluación y Acreditación de la Educación Superior (SICEVAES)}

El SICEVAES evalúa programas de Educación Superior de nivel de pregrado, grado y postgrado, así como el desempeño global de Instituciones de Educación Superior. En el primer ciclo de evaluaciones, de manera voluntaria, 98 programas de grado han iniciado procesos de autoevaluación. Asimismo, 6 universidades de la región han iniciado autoevaluación de su gestión, administración y desempeño institucional.

\section{INICIATIVAS ESPECÍFICAS EN EVALUACIÓN Y ACREDITACIÓN DE LA CALIDAD EN EDUCACIÓN SUPERIOR A DISTANCIA}

En el contexto norteamericano los organismos que tienen en cuenta la evaluación y acreditación de la Educación a Distancia también son muy escasos, entre ellos destacamos: "The Distance Education and Training Council" (DETC), que es una agencia acreditadora en el ámbito de la EaD, y está ubicada en los Estados Unidos de Norteamérica http://www.detc.org/about.html. Esta agencia tiene miembros acreditados en Canada, Ireland, Japan, South Africa, the United Kingdom, and the United States.

En el ámbito europeo, podemos citar a la EADTU (European Association of Distance Teaching Universities) que está trabajando a través del proyecto "Eexcellence" en estándares de excelencia en e-learning

Destacamos, también, por su aporte específico, a la Agencia Nacional de Evaluación de la Calidad y Acreditación Española, ANECA, quien, bajo el patrocinio del Ministerio de Educación y Ciencia tiene en el ámbito de sus competencias, entre otras, el de evaluar la Universidad Nacional de Educación a Distancia (UNED), encontrándose con las dificultades propias al tener que evaluar aspectos no comunes a otras universidades. Esta situación condujo a la creación de un grupo de trabajo en el que participó la propia UNED, para revisar y adecuar la Guía de evaluación de titulaciones a un modelo de enseñanza a distancia.

En el contexto de Latinoamérica y del Caribe, además de las pequeñas experiencias señaladas en apartados anteriores, se han desarrollado diferentes proyectos y convocado actividades diversas con la finalidad de avanzar en el desarrollo de la calidad y la certificación de la misma en busca de una acreditación nacional y/o internacional. Destacamos a continuación, según nuestro criterio, las más significativas: 
María José Rubio y Luis Miguel Romero

Apostar por la CAlidad de la EduCACión SuPERIor a Distancia desde la Pertinencia Social

\section{"Centro Virtual para el Desarrollo de Estándares de Calidad para la Educación Superior a Distancia en América Latina y el Caribe" www. utpl.edu.ec/centrovirtual}

El Proyecto "Centro Virtual para el Desarrollo de Estándares de Calidad para la Educación Superior a Distancia en América Latina y el Caribe" comenzó a gestarse con la preocupación del Banco Interamericano de Desarrollo (BID) y la invitación a una reunión de expertos, en Washington en agosto del año 2000, para analizar la situación de la Educación a Distancia en América Latina y el Caribe.

Desde ese momento se establecieron los contactos pertinentes entre la UTPL, el BID, la AIESAD y el CREAD, cristalizando, después de la reunión mantenida en Washington por los representantes de estas instituciones el 29 de mayo de 2002, el compromiso de todas las partes para llevar adelante el Programa.

El objetivo general del Proyecto fue el desarrollo de las bases para un sistema de acreditación y estándares de calidad para programas de educación superior a distancia en América Latina y el Caribe (Centro Virtual), y la realización de una validación preliminar de dichas bases mediante consultas y pruebas piloto. Entre los objetivos específicos se señalaron el promover que las instituciones de enseñanza superior puedan mejorar, lanzar y administrar con éxito, programas de educación a distancia basados en la tecnología de la información, y contribuir a la capacidad de los gobiernos para regular, evaluar y acreditar sus programas educativos a distancia.

Con el fin de perfeccionar y asegurar un sólido apoyo hacia el modelo de evaluación, se revisó y consolidó el mismo en una Reunión Técnica, en la cual los expertos, el Equipo Académico, el Comité Coordinador y Especialistas internacionales discutieron el contenido del documento de trabajo, se sugirieron modificaciones al mismo, y se aprobó el documento para su publicación posterior. La mayor parte de las discusiones se concentraron en los estándares de calidad propuestos en el documento de trabajo revisado, los cuales fueron los catalizadores para los intercambios en línea posteriores entre especialistas en educación a distancia de Iberoamérica y el Caribe. Los estándares inicialmente propuestos fueron modificados, como producto de esta reunión.

La importancia fundamental de este proyecto fue el consenso en él y la asimilación del mismo por un gran número de entidades y expertos al tener en cuenta los aspectos específicos que preocupaban a las universidades de la región que imparten EaD. Este trabajo se asumió como un reto de futuro pues se reflexionó ampliamente sobre el tema, considerando estándares mínimos aceptables para manejarnos en esta 
modalidad con confianza mutua, se acordó utilizar un lenguaje común para hablar de estándares y se tuvo en cuenta la experiencia de las instituciones de educación a distancia, lo cual, con el tiempo ha seguido dando sus frutos con la adaptación del modelo en diversas universidades participantes.

Como resultado del proyecto mencionado y las pruebas piloto realizadas actualmente existe una versión final del Centro Virtual a disposición de instituciones, gobiernos y educadores en el campo de la educación a distancia. En dicho Centro se puede realizar una valoración de cada estándar y además pueden comparar sus valoraciones con las que han obtenido otras instituciones. (www.utpl.edu.ec/ centrovirtual/)

Actualmente la página Web ha sido mejorada, se encuentra funcionando debido a una decisión institucional y gracias al mantenimiento que se le da por parte del equipo técnico de la UTPL. Este centro virtual está activo y cuenta con referencias en torno a la valoración de los estándares. Estos no son estáticos sino que nos irán dando razón de las tendencias en la calidad de la EaD.

Una vez terminado el proyecto y visto el interés del mismo se han generado espacios de reflexión en numerosos eventos sobre $\mathrm{EaD}$ que han requerido la presentación del proyecto en Encuentros, Seminarios y Congresos Internacionales, así como en las aplicaciones en diversas universidades.

\section{Avances del Proyecto Centro Virtual (Indicadores e Instrumentos de Evaluación)}

La propuesta del Centro Virtual que ya de por si fue excesivamente ambiciosa, exigía, una vez terminado el Proyecto para tener su máxima utilidad, precisar los indicadores, elaborar instrumentos de medida y evaluación. Por ello el equipo de evaluación de Modalidad a Distancia de la UTPL continuó trabajando el Proyecto a través de:

1. El análisis de estándares e indicadores

2. Selección de técnicas

3. Diseño de instrumentos para la recogida de información

4. Análisis de las valoraciones asignadas por distintos modelos y propuestas de ponderación por criterios, subcriterios, objetivos y estándares. 
María José Rubio y Luis Miguel Romero

Apostar por la Calidad de la Educación Superior a Distancia desde la Pertinencia Social

5. Elaboración del Registro de Autoevaluación

6. Redacción de las pautas para la preparación del proceso de autoevaluación

7. Publicación de diez documentos de trabajo, que detallan los aspectos anteriores: "Proceso de autoevaluación de los programas de Educación a Distancia, basado en el proyecto Centro Virtual para el Desarrollo de Estándares de Calidad para la Educación Superior a Distancia en América Latina y el Caribe", compuesto por un documento introductorio y un documento por cada uno de los 9 criterios.

8. La mejora y mantenimiento de la página del Centro Virtual

9. La elaboración de un portafolio electrónico, que permitirá registrar el proceso de autoevaluación de manera automatizada e incorporar la documentación de respaldo en formato digital.

Es de destacar igualmente la amplia difusión que se ha dado al proyecto, no sólo a través de las presentaciones del mismo, sino a través de la publicación de la Memoria, el envío de la misma a los organismos de evaluación y acreditación de Latinoamérica y el Caribe y a las universidades de la zona interesadas en educación a distancia. De igual forma se ha difundido a un gran número de especialistas en $\mathrm{EaD}$ a través de cursos de masterados y de expertos universitarios, en los que se ha incluido el tema de estándares de calidad (Universidad Católica del Perú, UTPL, Utem Virtual de Chile, Universidad de Sevilla), en asesorías a instituciones universitarias interesadas en la calidad de la educación a distancia (Universidades de Perú, Chile, Paraguay, México, Venezuela, Panamá, Ecuador, República Dominicana, Bolivia, Argentina), en foros virtuales a nivel internacional, en varios programas de la Televisión Iberoamericana y, a través de las redes de las asociaciones y entidades vinculadas (Consorcio Red de Educación a Distancia (CREAD), Asociación Iberoamericana de Educación Superior a Distancia (AIESAD), Red Global de Aprendizaje para el Desarrollo del Banco Mundial...)

Proyecto Alfa Rueda "Evaluación de los Programas de Educación a Distancia (Contrato No. AML/B7-311/97/o666/II-o153-A) www. javeriana.edu.co/cau/rueda

La Comunidad Europea dentro del programa ALFA - América Latina Formación Académica - auspició la conformación de la Red de Universidades de Educación a Distancia - RED RUEDA- con el fin de desarrollar el PROYECTO EVALUACIÓN DE LOS PROGRAMAS DE EDUCACIÓN A DISTANCIA durante el período comprendido entre noviembre de 2002 y julio de 2005. 
María José Rubio y Luis Miguel Romero

Apostar por la CAlidad de la Educación Superior a Distancia desde la Pertinencia Social

Las seis instituciones socias de este proyecto son las universidades latinoamericanas: UNIVERSIDAD TÉCNICA PARTICULAR DE LOJA (UTPL), Ecuador, PONTIFICIA UNIVERSIDAD JAVERIANA (PUJ), Colombia y UNIVERSIDAD NACIONAL ABIERTA (UNA), Venezuela y las universidades europeas: UNIVERSIDAD NACIONAL DE DUCACIÓN A DISTANCIA (UNED), España, UNIVERSITEIT GENT (UG), Bélgica y UNIVERSITÁ TELEMATICA GUGLIELMO MARCONI (UTGM), Italia.

Durante el desarrollo del proyecto se ha logrado crear y fortalecer un trabajo en red entre las instituciones participantes, la creación y edición de un instrumento de evaluación, validado en primera instancia mediante la aplicación en cada una de las seis universidades socias. Dicho instrumento se encuentra en una versión digital para facilitar su uso y fácil acceso.

Desafortunadamente y a pesar de que el Proyecto Alfa toma estándares del Proyecto Centro Virtual de Estándares de Calidad para la Educación Superior a Distancia en América Latina y el Caribe, no se consiguió que fuera la base para nuevos avances en el mismo y, por lo tanto, se perdieron grandes esfuerzos por tener en cuenta también diversas fuentes e iniciar de nuevo. Con ello la repercusión de ambos proyectos, en vez de sumarse se reduce. En el caso concreto del Proyecto Alfa, al igual que ha sucedido con otros muchos proyectos se quedan en eso, en proyectos, o en pequeñas aportaciones que hacen parte de la literatura de la historia con una mínima repercusión en experiencias de alguna de las instituciones participantes, todo ello porque no hay quien de continuidad al trabajo realizado.

\section{Congreso de Calidad en Educación a Distancia}

En el ámbito latinoamericano se han desarrollado varios eventos sobre la calidad en $\mathrm{EaD}$, lo que nos demuestra el interés que sobre el tema existe en la región; pero por sus consecuencias, creemos oportuno destacar el Congreso sobre Calidad y Acreditación Internacional en Educación Superior a Distancia - I Congreso CREAD Andes en el XV Aniversario del CREAD y I Encuentro Iberoamericano Virtual Educa sobre Acreditación en Educación Superior, celebrado en octubre del 2005 en la Universidad Técnica Particular de Loja (Ecuador), y que tuvo como base temática principal, el Proyecto Centro Virtual de Estándares de Calidad para la Educación Superior a Distancia en América Latina y el Caribe. El Congreso concentró a un gran número de instituciones y participantes y, dio lugar, a la creación del Instituto Latinoamericano y del Caribe de Calidad en Educación Superior a Distancia (CAL-ED). 
María José Rubio y Luis Miguel Romero

Apostar por la CAlidad de la EduCACión SuPERIor a Distancia desde la Pertinencia Social

Los objetivos del congreso fueron: Reflexionar sobre la calidad en Educación a Distancia desde sus múltiples perspectivas. Contribuir a la mejora de la calidad en Educación a Distancia a través de propuestas para la aplicación de estándares e indicadores. Proponer una opción de Acreditación Internacional de los procesos específicos de los Sistemas de Educación a Distancia.

Las áreas temáticas que se abordaron en el mismo fueron:

1. La calidad como reto en educación a distancia

2. Estándares e indicadores de calidad en educación a distancia

3. Acreditación internacional

\section{Instituto Latinoamericano y del Caribe de Calidad en Educación Superior a Distancia (CAL-ED) www.utpl.edu.ec/caled ${ }^{7}$}

Como hemos señalado uno de los logros más significativos del Congreso de Calidad en Educación a Distancia, en el que coincidieron los representantes de las instituciones y organismos más importantes de la $\mathrm{EaD}$, fue la creación del Instituto Latinoamericano y del Caribe de Calidad en Educación Superior a Distancia (CALED).

El CAL-ED se constituyó el 19 de octubre del 2005. Su misión principal es contribuir al mejoramiento de la calidad en la enseñanza superior a distancia en todas las instituciones de América Latina y el Caribe que ofrezcan este tipo de estudios.

Las instituciones que firmaron el acta de constitución de este Instituto en la ciudad de Loja (Ecuador), entendieron la necesidad de unir esfuerzos institucionales con el objetivo de promover y fortalecer la capacidad de las Universidades Latinoamericanas y del Caribe en la consecución de una oferta de Educación a Distancia de Calidad y tuvieron presente el importante papel que desempeña la ESaD en los procesos de desarrollo de los países Latinoamericanos y del Caribe, cumpliendo con ello con el principio de la UNESCO de Educación de calidad al alcance de todos.

Asumieron la necesidad manifestada de mejorar la calidad de los programas a distancia y reafirmaron la Propuesta de Estándares de Calidad en la Educación Superior a Distancia y las conclusiones especificadas de la Memoria del proyecto "Centro Virtual para el Desarrollo de Estándares de Calidad para la Educación Superior a Distancia en América Latina y el Caribe"8 y a su vez consideraron la autonomía universitaria y la diversidad cultural como principios básicos y elementos 
María José Rubio y Luis Miguel Romero

Apostar por la Calidad de la Educación Superior a Distancia desde la Pertinencia Social

a tener en cuenta en los procesos de evaluación y acreditación. Reconocieron la potestad de las agencias nacionales de evaluación de la calidad de los países de América Latina y el Caribe para acreditar las titulaciones y programas académicos de Educación Superior en sus respectivos países y acordaron los siguientes objetivos para el CAL-ED:

- Promover la cultura de la evaluación y de la calidad en la Educación Superior a Distancia.

- Contribuir a la elaboración de directrices e instrumentos para la evaluación, acreditación y certificación de Titulaciones, Programas y Servicios de Educación Superior a Distancia.

- Generar mecanismos de reconocimiento de la calidad basados en criterios, indicadores y estándares comunes para la Educación Superior a Distancia.

- Asesorar a las Universidades sobre los procesos de evaluación de la calidad y acreditación en Educación Superior a Distancia de América Latina y el Caribe.

- Coordinar esfuerzos y colaborar con las agencias nacionales de acreditación en los sistemas de Educación Superior a Distancia.

Es de destacar que para la creación del CAL-ED se unieron la mayoría de las instituciones preocupadas por la calidad en la educación a distancia, quedando conformado de la siguiente manera:

- Junta Directiva conformada por las entidades promotoras (CREAD, Consorcio Red de Educación a Distancia, AIESAD, - Asociación Iberoamericana de Educación Superior a Distancia, VIRTUAL EDUCA Y UTPL - Universidad Técnica Particular de Loja), quien como Secretaría Ejecutiva aplicará las orientaciones y recomendaciones propuestas por el Consejo Asesor y los Consejos Académicos.

Consejo Asesor: OEA (Organización de los Estados Americanos), OEI (Organización de Estados Iberoamericanos para la Educación, la Ciencia y la Cultura), ISTEC (Ibero American Science \& Technology Education Consortium), UDUAL (Unión de Universidades de América Latina y el Caribe), OUI (Organización Universitaria Interamericana), ICDE-LAC (International Council for Open and Distance Education - Latin América), IESALC-UNESCO (Instituto Internacional para la Educación Superior en América Latina y el Caribe) se excuso de no pertenecer a este Consejo en razón de sus reglamentos pero si existe la disposición a colaborar activamente con él. A éstas instituciones podrán sumarse otras, que asuman los objetivos del Instituto. Su función será asesorar a la Junta Directiva. 
María José Rubio y Luis Miguel Romero

Apostar por la Calidad de la Educación Superior a Distancia desde la Pertinencia Social

\section{Consejos Académicos:}

Universidades de Latinoamérica y del Caribe: UNAM (Universidad Nacional Autónoma de México), UNED-CR (Universidad Estatal a Distancia de Costa Rica), UNAD (Universidad Nacional a Distancia, Colombia), PUCP (Pontificia Universidad Católica del Perú), UCN (Universidad Católica del Norte Chile), IUA (Instituto Universitario Aeronáutico, Argentina), CEDERJ (Fundação Centro de Ciências e Educação Superior a Distância do Estado do Rio de Janeiro-Brasil), UNISUL (Universidade do Sul Santa Catarina, Brasil), UAPA (Universidad Abierta para Adultos, República Dominicana).

Universidades No Latinoamericanas y del Caribe: Cátedra UNESCO ED (UNED España), Cátedra UNESCO e-l (UOC -España), UNED-Es (Universidad Nacional de Educación a Distancia), UOC (Universidad Oberta de CatalunyaEspaña), EADTU (European Association of Distance Teaching Universities, Europa), NOVA (Nova Southeastern University - USA), PENN S (Penn State University USA), SUNY (State University of New Cork at Stoney Brook, USA), Acadia University (Canadá)

Entre las actividades iniciales que se han llevado a cabo en el CAL-ED destacamos las siguientes:

- Crear y mantener actualizada la página Web con información sobre calidad y acreditación en ESaD y, crear vínculos con otras páginas Web de interés para la evaluación y acreditación de la educación a distancia

- Trabajar en el consenso de estándares de calidad a través del "Centro Virtual para el Desarrollo de Estándares de Calidad para la Educación Superior a Distancia en América Latina y el Caribe".

- Servir de soporte al funcionamiento del "Centro Virtual para el Desarrollo de Estándares de Calidad para la Educación Superior a Distancia en América Latina y el Caribe" y continuación natural del mismo ya que se asumieron en su creación las claves de este proyecto. Así, además del Centro Virtual se ofrece un portafolio electrónico, con ejemplos, directrices e instrumentos de autoevaluación, que permite registrar el proceso de autoevaluación de manera automatizada e incorporar la documentación de respaldo en formato digital.

- Asesorar y acompañar en los procesos y puesta en marcha de sistemas a distancia a 20 universidades de 10 países diferentes, continuando la labor que ya se venía haciendo en la UTPL. 
- Ofrecimiento de cursos sobre principios básicos de la educación a distancia (-Fundamentos de la educación a distancia, - Universidad bimodal. Implementación del sistema de educación a distancia).

- Ofrecimiento de cursos sobre calidad en la educación a distancia (- Evaluación de la calidad en la educación a distancia, - Calidad de la docencia en Ead, Acompañamiento a la autoevaluación )

- Colaborar con las agencias nacionales e internacionales en la elaboración de directrices o Guías de autoevaluación con fines de acreditación para la ESaD. (Consejo Nacional de Evaluación y Acreditación (CONEA), Ecuador, Ministerio de Educación de Republica Dominicana, participación en seminarios convocados por Riaces y colaboración con EADTU (European Association of Distance Teaching Universities) en este caso con el fin de poner en común los proyectos sobre evaluación de la calidad en EaD.

\section{REQUERIMIENTOS NECESARIOS ACTUALES PARA EL DESARROLLO DE LOS PROCESOS DE EVALUACIÓN Y ACREDITACIÓN DE LA EDUCACIÓN SUPERIOR A DISTANCIA EN AMERICA LATINA Y EL CARIBE}

Antes de hablar de los requerimientos, hemos de destacar las repercusiones que ha tenido el Proyecto "Centro Virtual de Estándares de Calidad para la Educación Superior a Distancia en América Latina y el Caribe” en torno a la evaluación de la educación a distancia, entre otros:

- La presentación en multitud de congresos y el interés de las diversas instituciones por el mismo.

- El ingreso al Centro Virtual por un gran número de personas para realizar la valoración de los estándares.

- La adaptación de los estándares por diversas universidades tales como: el Consorcio de Universidades del Perú, La Universidad Abierta para Adultos de República Dominicana, la Universidad Católica del Norte de Chile y la Universidad Técnica Particular de Loja entre otros.

- Asimismo, han sido tenidos en cuenta en el proyecto Alfa Rueda.

- Fueron la referencia principal en el Congreso de Calidad y Acreditación Internacional en Educación Superior a Distancia, desarrollado por la UTPL en Loja-Ecuador y en la propia creación del Instituto Latinoamericano y del Caribe de Calidad en Educación Superior a Distancia CAL-ED. 
María José Rubio y Luis Miguel Romero

Apostar por la CAlidad de la Educación Superior a Distancia desde la Pertinencia Social

- Ha recibido un continuo apoyo de las dos asociaciones más importantes de educación a distancia en los países de la Región, el Consorcio Red de Educación a Distancia (CREAD) y de la Asociación Iberoamericana de Educación Superior a Distancia (AIESAD), así como de Virtual Educa, Programa Adscrito a la Cumbre Iberoamericana de Jefes de Estado y de Gobierno.

A pesar de toda la difusión y amplia participación, no podemos olvidar la tendencia a la dispersión, pues como añadíamos anteriormente, se ha seguido investigando en multitud de sistemas de estándares por diversas instituciones, en vez de dar continuidad a lo que ya se tenía. Todo ello ha generado una dispersión y confusión, que es necesario tener en cuenta en este momento preciso para seguir potenciando el trabajo de estándares consensuado y conocido.

\section{PROPUESTA DESDE LA PERTINENCIA}

Por todo lo anterior, y desde el punto de vista de la pertinencia en este momento, creemos que la tendencia a generar diversos modelos de calidad en educación a distancia no va a ayudar en absoluto al desarrollo de la misma, tanto por la fragmentación como por la falta de continuidad, lo que se refleja en la escasa e inadecuada legislación en los países sobre el tema, como hemos expuesto.

Naturalmente, un desarrollo del CAL-ED, en el que se integran y participan varias instituciones que dan credibilidad y solidez al organismo, con la finalidad de atender los procesos de evaluación y acreditación especialmente vinculados a las nuevas tendencias de internacionalización, intercambios y programas conjuntos de educación a distancia, podría ser una vía adecuada y rápida de responder a las necesidades mencionadas. Este desarrollo incluye la creación de Comités de Expertos de la calidad en educación a distancia teniendo en cuenta instituciones de prestigio con experiencia en la misma, la creación de equipos de evaluadores vinculados al CAL-ED y considerar los elementos legales y convenios correspondientes para que la certificación dada por el CAL-ED tenga un valor otorgado por los organismos internacionales que lo avalan, y a la vez sea tomada en cuenta por los organismos nacionales de acreditación. Finalmente, la investigación sobre calidad precisa tanto de elementos de continuidad, como de futuras e integradas innovaciones que recojan la rica diversidad de nuestro entorno; esto haría del CAL-ED un organismo abierto y en continua evolución. 


\section{NOTAS}

1 Rubio Gómez, M.J. (2003). Memoria Centro Virtual para el Desarrollo de Estándares de Calidad para la Educación Superior a Distancia en América Latina y el Caribe. BID N. ATN/SF-7867-RG. Loja - Ecuador. Universidad Técnica Particular de Loja

2 Consejo Nacional de Acreditación CNA (2005): Indicadores para la autoevaluación con fines de acreditación de programas de pregrado en las modalidades a distancia y virtual. pp. 15-16. Disponible en: http://www.iesalc.unesco.org.ve/acreditacion/ colombia/guiasacrd-co/lin aut fin acr pro pre mod dis vir ver pre.pdf (consulta 2006, 24 de agosto)

3 Estructura y Titulaciones de Educación Superior en Cuba. Disponible en: http:// www.oei.org.es/homologaciones/cuba.pdf. p3 (consulta 2006, 24 de agosto)

4 Comités Interinstitucionales para la Evaluación de la Educación Superior (CIEES). Disponible en: www.ciees.edu.mx (consulta 2006, 19 de octubre)

5 Sánchez, D. Yánez, J. Martínez, V: (2005): UNADP: Un Espacio de Convergencia Nacional. Disponible en: http://somi.cinstrum.unam.mx/virtualeduca2005/ resumenes/2005-03-17150PONENCIA.doc (consulta 2006, 25 de agosto).

6 Marrero, A. y Barros, G: Estructura y Titulaciones de Educación Superior en Uruguay. Disponible en: http://www.oei.org.es/homologaciones/uruguay.pdf\#search=\%22ley \%20de\%20educaci\%C3\%B3n\%20superior\%20uruguay\%22 (consulta 2006, 25 de agosto)

7 CAL-ED (2006): Creación y puesta en marcha del CAL-ED. Loja - Ecuador. Universidad Técnica Particular de Loja

8 Rubio Gómez, M.J. (2003): "Centro Virtual para el Desarrollo de Estándares de Calidad para la Educación Superior a Distancia en América Latina y el Caribe”.

\section{REFERENCIAS BIBLIOGRÁFICAS}

CAL-ED (2006): Creación y puesta en marcha del CAL-ED. Loja - Ecuador. Universidad Técnica Particular de Loja. Disponible en: www.utpl.edu.ec/caled [consulta 2006, 19 de octubre]

Comités Interinstitucionales para la Evaluación de la Educación Superior (CIEES). Disponible en: www.ciees. edu.mx [consulta 2006, 19 de octubre]

Consejo Nacional de Acreditación CNA (2005): Indicadores para la autoevaluaciónconfinesdeacreditación de programas de pregrado en las modalidades a distancia y virtual. pp 15-16. Disponible en: http://www. iesalc.unesco.org.ve/acreditacion/ colombia/guiasacrd-co/lin aut fin acr pro pre mod dis vir ver pre. pdf [consulta 2006, 24 de agosto]

Estructura y Titulaciones de Educación Superior en Cuba. Disponible en: http:// www.oei.org.es/homologaciones/cuba. pdf. [consulta 2006, 24 de agosto]

Evaluación de los Programas de Educación a Distancia Proyecto Alfa Rueda (Contrato No. AML/B7-311/97/0666/ II-0153-A) Disponible en: www. javeriana.edu.co/cau/rueda [consulta en 2006, 25 de agosto]

Marrero, A. y Barros, G: Estructura y Titulaciones de Educación Superior en 
María José Rubio y Luis Miguel Romero

Apostar por la CAlidad de la Educación Superior a Distancia desde la Pertinencia Social

Uruguay. Disponible en: http://www. oei.org.es/homologaciones/uruguay. pdf\#search $=\% 22$ ley\%20de\%20educac i\%C3\%B3n\%20superior\%20uruguay\% 22 [consulta 2006, 25 de agosto]

Rubio Gómez, M.J. (2003). Memoria Centro Virtual para el Desarrollo de Estándares de Calidad para la Educación Superior a Distancia en América Latina y el Caribe. BID N. ATN/SF-7867-RG. Loja - Ecuador. Universidad Técnica Particular de Loja
Sánchez, D. Yánez, J. Martínez, V: (2005): UNADP: Un Espacio de Convergencia Nacional. Disponible en: http://somi.cinstrum.unam.mx/ virtualeduca2005/resumenes/200503-17150PONENCIA.doc (consulta 2006, 25 de agosto).

The Distance Education and Training Council (DETC). Disponible en: http:// www.detc.org/about.html (consulta 2006, 25 de agosto).

\section{PALABRAS CLAVE}

Educación superior a distancia, Pertinencia, Reglamentación, Calidad, Acreditación, Estándares

\section{KEYWORDS}

Distance Higher Education, Relevance, Regulations, Quality, Accreditation, Standards

\section{PERFIL ACADEMICO DE LOS AUTORES}

María José Rubio Gómez, Dra. en Filosofía y CC. Ed, UNED-España. Del 88/97 Directora Centro UNED-Baleares. Actualmente: Directora Modalidad Distancia, Universidad Técnica Particular de Loja; miembro Comisión especial de Educación a Distancia y Virtual, Consejo Nacional de Evaluación y Acreditación del Ecuador (CONEA-Ecuador). Coordinadora Proyecto "Centro Virtual para el Desarrollo de Estándares de Calidad”, auspiciado por el BID y otros en evaluación de la calidad de la educación a distancia.

Dirección Postal: $\quad$ Universidad Técnica Particular de Loja. Ap. 11-01-608. LojaEcuador e-mail: mjrubio@utpl.edu.ec 
Luis Miguel Romero Fernández, Licenciado en Biología y Filosofía, Doctor en Medicina por la Universidad de Zaragoza, España. Rector-Canciller de la Universidad Técnica Particular de Loja (Ecuador). Miembro de los consejos directivos de AIESAD, CREAD, y OUI. Director del Instituto Latinoamericano de Calidad en Educación Superior a Distancia (CALED).

DirecciónPostal: $\quad$ Universidad Técnica Particular de Loja. Ap. 11-01-608. LojaEcuador

e-mail: lmromero@utpl.edu.ec

Fecha de entrega: 23.10.06

Fecha de aceptación: 20.12.06 